\title{
Stability Measurement of Ion Beam Current from Argon Field Ion Source*
}

\author{
Ryuta Okawa and Yuuki Morikawa \\ Graduate School of Engineering, Mie University, \\ 1577 Kurima-machiya-cho, Tsu 514-850\%, Japan
Graduate School of Engineering, Mie University, 1577 Kurima-machiya-cho, Tsu 514-8507, Japan and Center for Ultimate Technology on nano-Electronics, Mie University, 1577 Kurima-machiya, Tsu 514-8507, Japan \\ Shigekazu Nagai, Tatsuo Iwata, Kazuo Kajiwara, and Koichi Hata ${ }^{\dagger}$ \\ (Received 26 July 2011; Accepted 29 August 2011; Published 1 October 2011)
}

\begin{abstract}
We have been developing a next generation finely focused ion beam (FIB) system with noble gas field ion source (GFIS), which could reduce the contamination of irradiated ion species. Ion current stability of GFIS is one of the most fundamental factors for FIB applications. In this study, we report a preliminary measurement of current stability of field-ionized $\mathrm{Ar}^{+}$ions from a tungsten emitter-tip which has a specific nanoprotrusion terminated by a trimer on the tip-apex. As a measure of long-term stability of $\mathrm{Ar}^{+}$ion beam, current fluctuation of $\sim 5 \%$ for 1800 seconds was typically obtained at the beam current of $1.3 \mathrm{pA}$ and the argon gas pressure of $5 \times 10^{-3} \mathrm{~Pa}$. Furthermore it was found in argon-GFIS that the spike-like current fluctuation was correlated with the trimer-dimer transformation in a tungsten trimer-tip. [DOI: 10.1380/ejssnt.2011.371]
\end{abstract}

Keywords: Field ion microscopy; Field evaporation; Field ionization; Ion emission

\section{INTRODUCTION}

In modern nanotechnology fields, focused ion beam (FIB) instruments equipped with a gallium liquid metal ion source (Ga-LIMS) have been widely used for the nanoprocessing, including sample preparation for transmission electron microscopy and nano-size repairing of interconnects in large-scale integrated circuits. At the present time, electrical contamination with irradiated $\mathrm{Ga}$ ions becomes more serious as the design rule ultimately reaches to several tens of nanometers. To overcome the contamination problem, one of the expectable ion sources is the noble gas field ion source (GFIS) [1]. However it is well known that the ion current emitted from GFIS is far smaller than that of LMIS. In the case of GFIS, therefore, the higher angular current density and heavier noble gas-ions should be required for FIB applications.

In our earlier study, it was confirmed that the smaller the taper angle of emitter-tip is, the larger the capture area of gas ions $[2,3]$. In order to more improve the angular current density of GFIS we have developed tungsten emitter-tips with a specific nanoprotrusion on the tip-apex by using a modified field-assisted oxygen etching, followed by a field evaporation process to fabricate a trimer on the nanoprotrusion [4]. Whereas, nitrogen etching process, in which a subnanometric protrusion such as a single tungsten atom could be formed on $\langle 110\rangle$ - or $\langle 111\rangle$ oriented crystalline tungsten emitter-tips $[5,6]$.

As far as we know, there is no detailed analysis of the long-term stability in respect to current density from argon-GFIS compared to those from helium- [7] and neonGFIS [8]. In this study, we report a preliminary measurement of current stability of argon-GFIS equipped with

*This paper was presented at 8th International Symposium on Atomic Level Characterizations for New Materials and Devices, Olympic Parktel, Seoul, Republic of Korea, 22-27 May, 2011.

$\dagger$ Corresponding author: hata@elec.mie-u.ac.jp
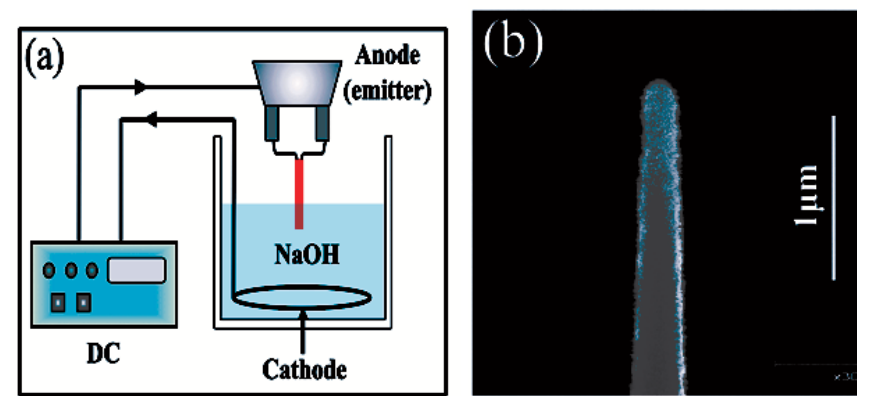

FIG. 1: (a) Schematic of the electrolytic polishing apparatus. (b) SEM image of an electrolytically polished emitter-tip.

a tungsten emitter-tip having a specific nanoprotrusion terminated with a trimer on the tip-apex, which was fabricated by a modified field assisted nitrogen etching and field evaporation process.

\section{EXPERIMENTAL PROCEDURES}

First, a $\langle 111\rangle$-oriented single-crystalline tungsten wire with a diameter of $0.127 \mathrm{~mm}$ was used for an emitter material. A tungsten wire was electrolytically polished in aqueous solution of $\mathrm{NaOH}(5 \mathrm{~mol} / \mathrm{L})$ at $50^{\circ} \mathrm{C}$ with an apparatus shown in Fig. 1(a). Figure 1(b) shows a scanning electron microscopy (SEM) image of a typical emitter-tip in the end of electrolytically-polishing process.

Secondly, an electrolytically-polished emitter-tip was introduced into a field-assisted nitrogen etching apparatus shown in Fig. 2. In the field-assisted nitrogen etching process, a specific nanoprotrusion was formed on the tip-apex to obtain a higher angular current density. We performed a modified method based on a field-assisted nitrogen etching, which was reported by Rezeq et al. and Onoda et al. in detail $[5,6]$. The nanoprotrusion with a hemispherical shape even along $\langle 111\rangle$ direction could be formed on the 


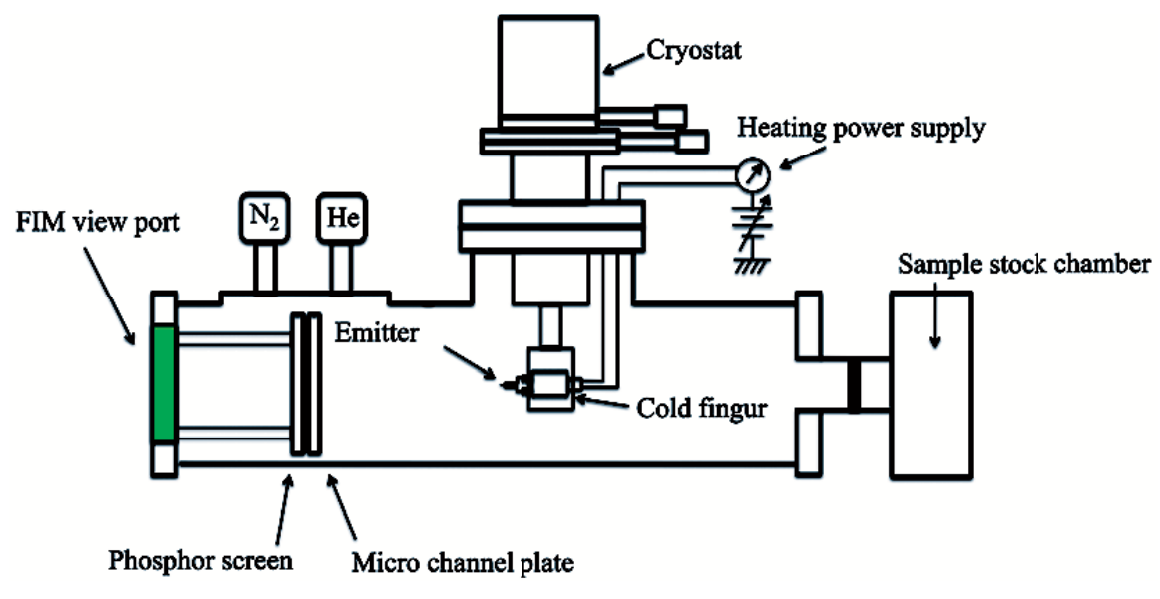

FIG. 2: Schematic of the field-assisted gas-etching apparatus.

tip-apex with a significantly large radius of curvature by the modified field-assisted nitrogen etching process.

Finally, the emitter-tip with a nanoprotrusion was introduced into a field ion microscope (FIM) apparatus equipped with a field emission microscope (FEM), which consisted of the emission-current measurement chamber, sample stock chamber, and gas purification chamber, as shown in Fig. 3. The attained pressure of measurement chamber was $2 \times 10^{-8} \mathrm{~Pa}$. The temperature of the emitter was cooled down to $20 \mathrm{~K}$ with the cryostat. The emitter temperature was kept constant in the range from $20 \mathrm{~K}$ to $300 \mathrm{~K}$ by using a feedback control circuit. In the measurement chamber, images of emitter-tip apex were observed by both FIM and FEM. A probe hole with half angle of $30 \mathrm{mrad}$ was placed at the center of a micro channel plate (MCP) for FIM. Field-ionized ions passed through the probe hole were collected by a Faraday cup, and the ion current was measured by a pico-ammeter (Keithley type-6485). After that, FIM movies were recorded by a commercial digital video camera.

\section{RESULTS AND DISCUSSION}

Sequential FIM images of a tungsten emitter-tip during the field-assisted nitrogen etching process are shown in Fig. 4. Figure 4(a) is a helium-FIM image of a tungsten emitter-tip as initially formed by field evaporation in helium ambient at the pressure of $1 \times 10^{-3} \mathrm{~Pa}$. Subsequently, $\mathrm{N}_{2}$ gas was additionally introduced up to $5 \times 10^{-4} \mathrm{~Pa}$, and the field-assisted nitrogen etching was performed. In Fig. 4(b), the central region was imaged by helium ions, whereas the peripheral ring-like one was due to nitrogen ions. When the etching process reached to the stage in which a specific nanoprotrusion was formed on the tungsten emitter-tip, as shown in Fig. 4(c), the supply of $\mathrm{N}_{2}$ gas was stopped. In the final process, the nanoprotrusion was terminated with three tungsten atoms (i.e., trimer) by field evaporation, as shown in Fig. 4(d).

Next, the emitter-tip was transferred to the measurement chamber of the FIM-FEM apparatus. By a careful field evaporation treatment in helium ambient of $1 \times 10^{-3} \mathrm{~Pa}$, the emitter-tip terminated with the simi- lar trimer as that in Fig. 4(d) was obtained as shown in Fig. 5(a). In order to measure the field-ionized $\mathrm{Ar}^{+}$ion current, the applied voltage was down to ionization field of argon and ambient gas was changed from helium to argon. Figure 5(b) shows the FIM image in argon imaging gas at $5 \times 10^{-5} \mathrm{~Pa}$. The resolution of argon-FIM image is lower than that of helium one, as shown in Figs. 5(a) and 5 (b), since the lower the emitter-tip temperature is and the higher the ionization field of an imaging gas is, the better the resolution of FIM image. The measurement of ion current through the probe hole was performed during 1800 seconds at argon gas pressure of $5 \times 10^{-3} \mathrm{~Pa}$. As a result, $\mathrm{Ar}^{+}$ion current with a fluctuation of $5.4 \%$ during 1800 seconds was obtained at average current of $1.3 \mathrm{pA}$, as shown in Fig. 5(c). In this case, the angular current density was estimated to be $1.5 \mathrm{nA} / \mathrm{sr}$. Furthermore, it was found that a number of spike-like fluctuations occurred frequently, as indicated by arrows in Fig. 5(c).

In order to elucidate the cause of their spike-like fluctuations, we recorded a FIM movie by a digital video camera. As a result, it was confirmed that the trimer shown in Fig. 6(a) transformed promptly to one dimer among their three configurations as shown in Figs. 6(b)(d), and returned immediately to the trimer as before. This transformation between the quasi-stable trimer and one of the three unstable dimers occurred repeatedly during the whole time of video-camera recording. It is speculated that the spike-like fluctuations was correlated closely with the phenomenon of the trimer-dimer transformation.

We could give a plausible hypothesis as follows, which is explicable to the phenomenon of trimer-dimer transformation abovementioned. $\mathrm{Fu}$ et al. report that in the atomic processes of 29 tungsten-atoms cluster on a W (111) terrace, both step-down and step-up motions occur at the temperature of $\sim 650 \mathrm{~K}$ with no electric field [9], that is, only the thermal energy of $\sim 56 \mathrm{meV}$ gives rise to their atomic processes. In our experiment, the topmost layer consists of only three atoms (i.e. trimer), which are peripheral atoms. Therefore, the step-down motion gives rise to the transformation from trimer to dimer, and stepup motion gives rise to the transformation from dimer to trimer, probably due to the best image field $(\sim 22 \mathrm{~V} / \mathrm{nm}$ for $\mathrm{Ar}$ ) with thermal energy of at most $\sim 25 \mathrm{meV}$ (i.e. 


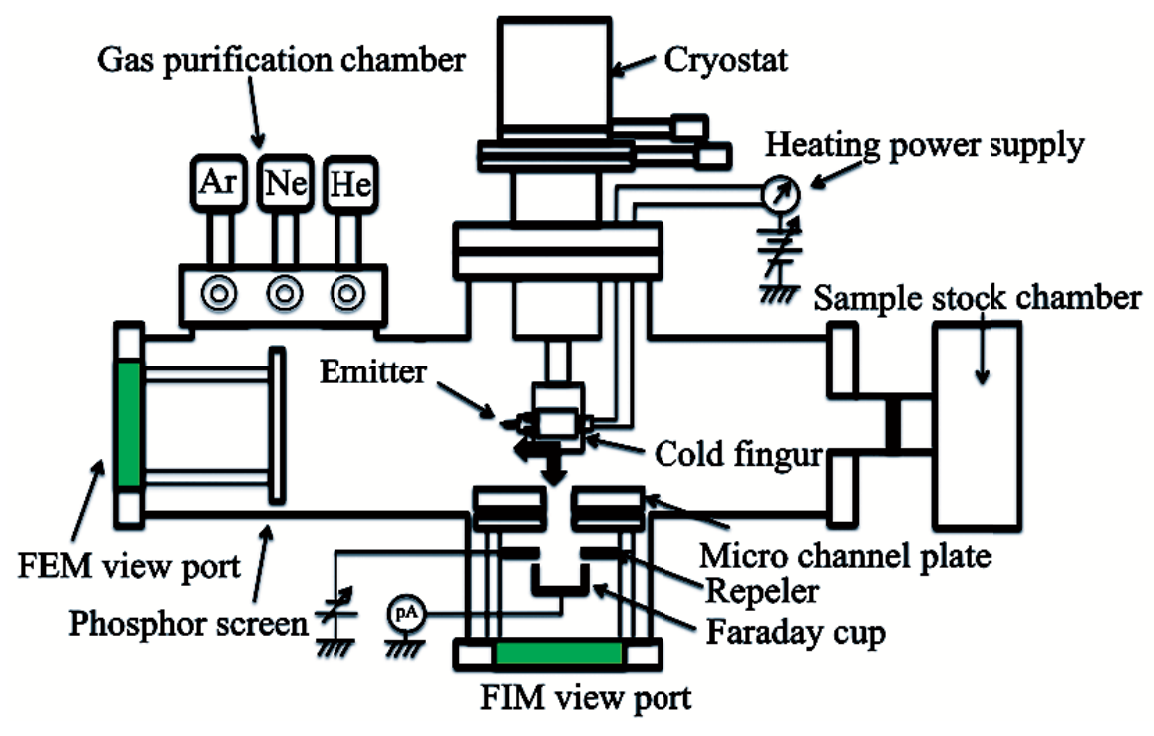

FIG. 3: Schematic of the FIM-FEM apparatus.
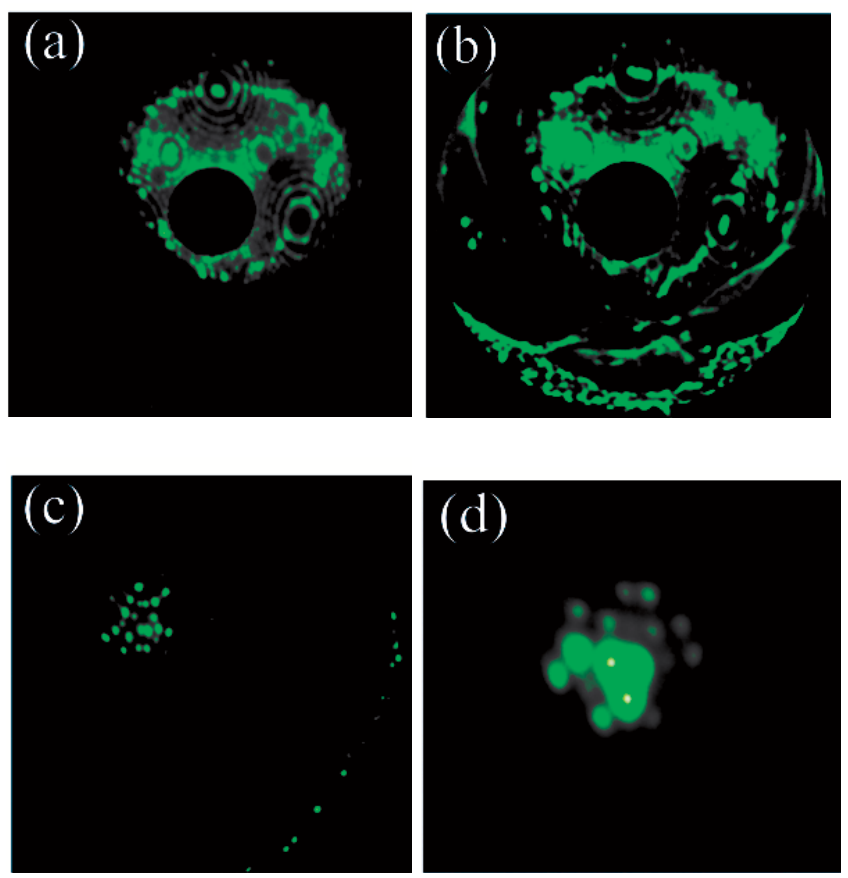

FIG. 4: (a) He-FIM image of a W emitter-tip as initially formed by field evaporation. He pressure was $1 \times 10^{-3} \mathrm{~Pa}$. Applied voltage $V=10 \mathrm{kV}$. Emitter temperature $T_{\mathrm{em}}=30 \mathrm{~K}$. (b)-(c) Sequential FIM images of the same tip during fieldassisted nitrogen etching. He pressure was $1 \times 10^{-3} \mathrm{~Pa} . \mathrm{N}_{2}$ pressure was $5 \times 10^{-4} \mathrm{~Pa}$. $V=10 \mathrm{kV} . T_{\mathrm{em}}=100 \mathrm{~K}$. (d) HeFIM image of a trimer on the tip-apex at the final stage. He pressure was $1 \times 10^{-3} \mathrm{~Pa} . V=9.5 \mathrm{kV} . T_{\mathrm{em}}=30 \mathrm{~K}$.

$$
\sim 300 \mathrm{~K})
$$

On the other hand, Tsong reports that the polarization energy $E_{\mathrm{k}}$ of image gas is supposed to be $1 / 2 \alpha F_{\mathrm{i}}^{2}$, where $\alpha$ is the gas-atom polarizability and $F_{\mathrm{i}}$ is the best-image field [10]. He also reports that during the accommodation process, which is the interaction between impinging gas
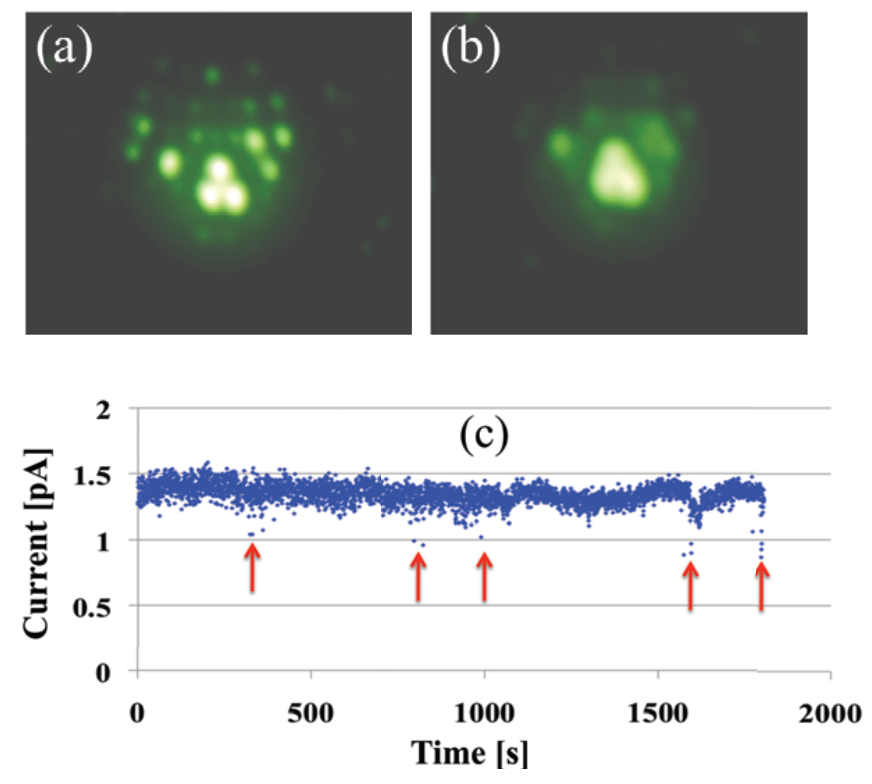

FIG. 5: (a) He-FIM image of the similar trimer-tip as that shown in Fig. 4. He pressure was $1 \times 10^{-3} \mathrm{~Pa} . V=9.5 \mathrm{kV}$. (b) Ar-FIM image of the same trimer shown in (a). Ar pressure was $5 \times 10^{-3} \mathrm{kV} . V=4.3 \mathrm{kV} . T_{\mathrm{em}}=90 \mathrm{~K}$. (c) $\mathrm{Ar}^{+}$ion current variation emitted from the trimer-tip. Average current was $\sim 1.3 \mathrm{pA}$. Angular current density was $\sim 1.5 \mathrm{nA} / \mathrm{sr}$.

atoms and surface atoms of tungsten emitter-tip, the energy exchange can be represented by the accommodation coefficient, $a$, which is given to be 0.315 for argon gas on Mo and 0.020 for helium gas on W, respectively [10]. In addition, Forbes reports that $E_{\mathrm{k}}$ values for argon and helium are $195 \mathrm{meV}$ and $138 \mathrm{meV}$, respectively [11]. Based on a crude calculation, the energy transfer is estimated to be $\sim 60 \mathrm{meV}$ for $\mathrm{Ar} / \mathrm{Mo}$ and $\sim 3 \mathrm{meV}$ for $\mathrm{He} / \mathrm{W}$ in a first hopping motion, respectively. Taking these roughly estimated values into account in $\mathrm{Ar} / \mathrm{W}$ case, the transferred energy is in good agreement with the thermal en- 

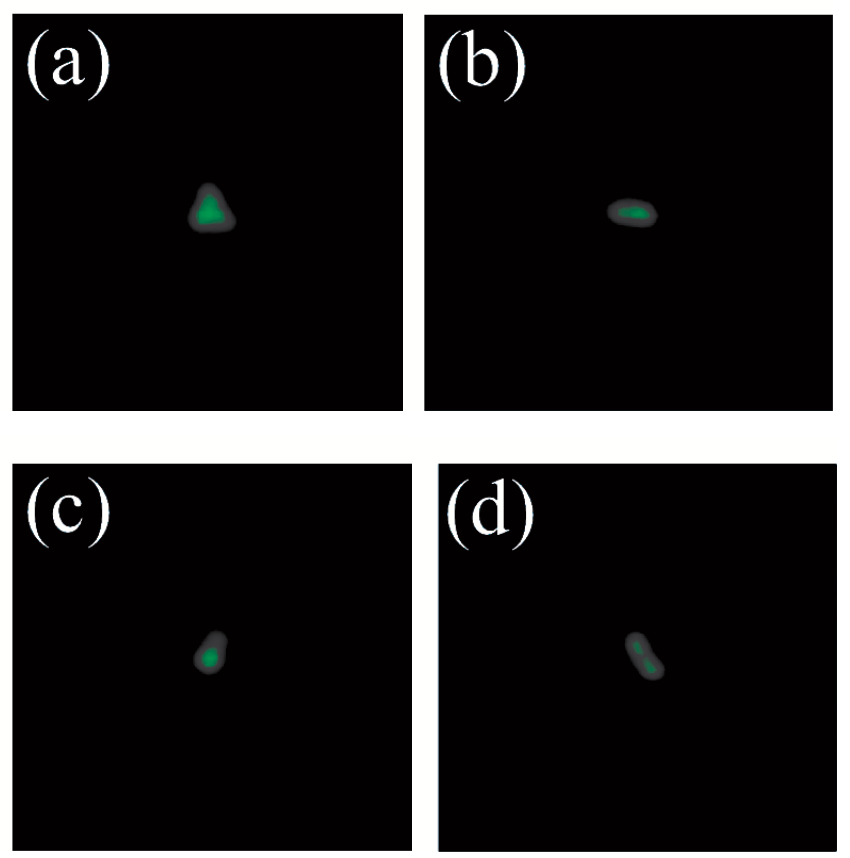

FIG. 6: A series of FIM images of the same trimer-tip as that shown in Fig. 5. The transformation between a quasistable trimer (a) and one of the three configurations of unstable dimers (b)-(d) was observed repeatedly. Ar pressure was $5 \times 10^{-3} \mathrm{~Pa}$.

ergy required for both step-up and step-down motions in the case of $\mathrm{W}$ clusters on W(111) terrace under the Fu's experimental conditions [9]. Therefore, it could be speculated that the trimer-dimer transformation on the topmost layer in argon-image gas probably occur more frequently than those in helium-image gas.

$\mathrm{Fu}$ et al. also report that the measured activation energies of diffusion $E_{\mathrm{d}}$ for the tungsten dimer and trimer on a $\mathrm{W}(111)$ terrace are $1.62 \mathrm{eV}$ and $1.74 \mathrm{eV}$, respec- tively [9]. Therefore, the difference of $E_{\mathrm{d}}$ between the dimer and trimer is $\sim 120 \mathrm{meV}$ in their experiment. This evidence could correlate to the difference of surface energy between the dimer- and trimer-geometrical structures. It suggests that the tungsten dimer is more unstable than the trimer, which could explain the quick recovery from dimer to trimer in our experiment, since the driving force is speculated to be the principle of minimizing the surface energy of geometrical structure.

In the near future, it should be necessary to clarify the behavior of trimer-dimer transformation to realize the FIB instruments equipped with argon-GFIS for the application to nanotechnology fields.

\section{CONCLUSIONS}

We performed, for the first time, a preliminary stability measurement of $\mathrm{Ar}^{+}$ion current as a measure of argon-GFIS, which was field-emitted from the tungsten emitter-tip having a specific nanoprotrusion terminated by a trimer on the tip-apex. As a result, long-term stability of argon ion beam, typically the current fluctuation of $\sim 5 \%$ for 1800 seconds, was obtained. It was also found that the transformation between the quasi-stable trimer and one of the three unstable dimers occurred repeatedly during the measurement. It was speculated that the spike-like fluctuation was correlated closely with the phenomenon of trimer-dimer transformation.

\section{Acknowledgments}

This work was supported by the national project, "Development of System and Technology for Advanced Measurement and Analysis", sponsored by the Ministry of Education, Culture, Sports, Science and Technology in Japan.
[1] V. N. Tondare, J. Vac. Sci. Technol. A 23, 1498 (2005).

[2] Y. Kobayashi, Y. Sugiyama, Y. Morikawa, K. Kajiwara, and K. Hata, Surf. Interface Anal. 42, 1544 (2010).

[3] Y. Sugiyama, Y. Kobayashi, Y. Morikawa, K. Kajiwara, and K. Hata, e-J. Surf. Sci. Nanotech. 8, 174 (2010).

[4] Y. Sugiura, H. Liu, T. Iwata, S. Nagai, K. Kajiwara, K. Asaka, Y. Saito, and K. Hata, Proc. 8th Int. Symp. on Atomic Level Characterization for New Materials and Devices, May 22-27, Seoul, Korea, 205 (2011).

[5] M. Rezeq, J. Pitters, and R. Wolkow, J.Chem. Phys. 124, 204716 (2006).

[6] J. Onoda, F. Rahman, and S. Mizuno, e-J. Surf. Sci. Nan- otech. 6, 152 (2008).

[7] B. W. Ward, J. A. Notte, and N. P. Economou, J. Vac. Sci. Technol. B 24, 2871 (2006).

[8] J. Notte, F. H. M. Faridur Rahman, S. McVey, S. Tan, and R. H. Livengood, Microsc. Microanal. 16, S2 28 (2010).

[9] T. Y. Fu, W. J. Weng, and T. T. Tsong, Appl, Surf. Sci. 254, 7831 (2008).

[10] T. T. Tsong, Atom-Probe Field Ion Microscopy (Cambridge Univ. Press, NY, 1990), p. 15.

[11] R. G. Forbes, Appl. Surf. Sci. 94/95, 1 (1996). 